\title{
Switching between Mii and Wii: The effects of cultural priming on the social affective $\mathbf{N} 400$
}

\author{
Michelle C. Fong • Sharon G. Goto • Colleen Moore • \\ Tracy Zhao $\cdot$ Zachary Schudson $\cdot$ Richard S. Lewis
}

Accepted: 2 May 2014/Published online: 28 May 2014

(C) Springer-Verlag Berlin Heidelberg 2014

\begin{abstract}
The effect of priming cultural schemas on the neural mechanisms underlying attention is not well understood. Individuals in multicultural environments often switch between cultural schemas according to the immediate sociocultural context (Hong et al. in Am Psychol, 55:709-720, 2000). Across behavioral and neural measures, individuals from collectivist cultures (e.g., East Asians) have been shown to allocate greater attention to background objects and are more sensitive to social context relative to individuals from individualist cultures (e.g., European Americans). We sought to examine whether or not priming of independent and interdependent self-construal affects neural activity underlying attention to social contexts in bicultural (i.e., East Asian Americans) and monocultural (i.e., European Americans) individuals. Using a modified N400 event-related potential design, we measured the degree to which East Asian American participants and European American participants, responded to affective incongruity in the emotional expression of a central figure relative to the surrounding figures. As predicted, when East Asian American bicultural participants were primed with interdependent values they displayed greater N400s to incongruent affective stimuli than congruent affective stimuli. In contrast, when primed with independent values, bicultural participants did not display a greater N400 to incongruent stimuli. European American participants did not display a greater N400 under either priming condition. Implications for the N400, biculturalism, and dynamic culture are discussed.
\end{abstract}

\section{Present Address:}

M. C. Fong $(\bowtie)$

Department of Psychology, University of Oregon, Eugene, OR 97403, USA

e-mail: mfong@uoregon.edu

M. C. Fong

Department of Humanities, Social Sciences, and Arts, Harvey Mudd College, Claremont, CA, USA

S. G. Goto · C. Moore - T. Zhao - Z. Schudson · R. S. Lewis

Department of Psychology, Pomona College, Claremont, CA, USA 
Keywords N400 - Affect · Context - Biculturalism - Dynamic culture · Collectivism

\section{Introduction}

The constructs of individualism and collectivism have played a central role in our understanding of culture (Markus and Kitayama 1999; Nisbett et al. 2001; Triandis et al. 1990). Fundamentally, cultural difference has been largely understood in terms of the ways the individual relates to important groups. For example, individualistic societies such as the United States value independence while collectivist societies such as East Asian nations value interdependence and see their behavior in relation to others (Nisbett et al. 2001). In this way, collectivism and individualism mediate the relation between culture and psychological processes (Hong et al. 2000; Markus and Hamedani 2007). This usage conceptualizes culture, and in particular individualism and collectivism, as mutually exclusive and relatively static constructs that change only across large spans of time due to affluence, modernity, and acculturation (Triandis 2000).

Although the focus on the individualism and collectivism constructs is longstanding, the conceptualization and methodology surrounding the constructs continually evolve to capture cultural diasporas and adaptation. Oyserman et al.'s (2002) meta-analysis used both international and domestic minority populations to better understand the relation between individualism and collectivism. This analysis revealed the factors to be more obliquely than inversely related to one another. The conceptualization of individualism and collectivism as orthogonal is consistent with Berry's (1980) bidimensional conceptualization of acculturation. In this framework, some individuals (i.e., biculturals) maintain both culture of origin and mainstream culture as opposed to privileging one and excluding the other (e.g., adopting an individualistic orientation to the exclusion of collectivism). This acculturative framework has gained much empirical support (Lee et al. 2006; Yoon et al. 2011).

As a possible mechanism for bicultural duality, conceptualizations of the individual possessing multiple and indeed, contrasting notions of the self is useful. Hong et al. (2000) put forth a model of culture as fluid in their dynamic constructivist approach. In a series of priming studies on bicultural Hong Kong Chinese samples, Hong et al. (2000) found that priming the salience of Chinese versus American cognitive schemas affected internal or external attributions. Chinese cultural primes resulted in external attributions, whereas American primes resulted in internal attributions. Other research using individualist and collectivist primes have resulted in a robust pattern of behavioral influences ranging from effects on the self (e.g., Trafimow et al. 1991), cognitive complexity (e.g., Tadmor et al. 2009), cultural values (e.g., Briley and Wyer 2002), and well-being (e.g., Chentsova-Dutton and Tsai 2010; Oishi 2000) (see Oyserman and Lee 2008 for an excellent review).

Recent studies employing neuroscientific techniques suggest that cultural priming also influences neural representations of the self. For example, Chiao 
et al. (2009) primed bicultural Asian Americans with individualistic and collectivistic values. Participants receiving the independent prime displayed increased activation of the medial prefrontal and posterior cingulate cortex during general relative to contextual self-judgments. Conversely, participants presented with the interdependent prime displayed greater activation for contextual relative to general self-judgments. Chiao et al. concluded that cultural priming influences brain activation associated with culturally congruent self-representations. In another study of Asian American biculturals, Harada et al. (2010) found that cultural priming influenced activation of the dorsal medial prefrontal cortex but not the ventral medial prefrontal cortex during evaluation of self-relevant information, suggesting that cultural priming may have a relatively specific influence on medial prefrontal brain activity.

Han and colleagues investigated self-construal priming in a series of studies using face presentations. This resulted in differential priming effects for interdependent and independent primes. For example, Sui and Han (2007) primed self-construal in Chinese participants during the presentation of faces of the self and a familiar other. Independent self-construal priming resulted in greater right frontal activity to self than to other, but no difference in activity was found following interdependent priming. In a sample of Westernized Chinese, Western priming increased ventromedial prefrontal cortex differentiation between the self and other, whereas Chinese priming decreased neural differentiation of the self and other ( $\mathrm{Ng}$ et al. 2010). In a third study, British participants who were interdependently primed showed reduced N2 amplitude to faces of the self relative to the no priming condition, but independent self construal priming had no effect (Sui et al. 2013). For the Chinese participants, however, independent selfconstrual priming reduced the $\mathrm{N} 2$ amplitude to friend's face relative to the no prime condition, whereas interdependent priming had no effect on the N2 amplitude to friend's face. In contrast, priming did not affect N2 amplitude to faces of the self. They concluded that when the priming condition was a mismatch with their 'chronic' cultural orientation, the culturally typical N2 effect was eliminated, whereas when the priming matched their chronic cultural orientation there was no influence on the $\mathrm{N} 2$.

Whereas most of the cultural priming studies have focused on neural representations of the self, Lin et al. (2008) investigated self-construal priming in Chinese participants during an ERP experiment requiring participants to discriminate local or global letters of compound local/global letter stimuli. Following independent self-construal priming, participants showed an increased P1 amplitude to local compared to global targets at occipital electrode sites. Following interdependent self-construal priming, participants showed increased P1 amplitude to global relative to local targets. They interpreted these data to reflect changes in early, extrastriate visual perceptual processing associated with self-construal priming.

Together, these data suggest that the pattern of neural effects is robust and wide ranging. Self-construal priming has been shown to affect visual processing of early ERP components (Lin et al. 2008; Sui et al. 2013) as well as frontal activation associated with self and other judgments (Chiao et al. 2009; Harada et al. 2010; Sui 
and Han 2007; Sui et al. 2013). However, it is unclear to what extent self-construal priming affects the broader range of cultural attributes. We sought to better understand and extend neurally primed effects by focusing on how individuals process socioaffective information.

Social information may receive differential attention as a function of culture. Theoretically, Nisbett et al. (2001) have conceptualized two types of cognitive processing styles: holistic and analytic cognition. Analytic processing styles are thought to emphasize the constancy of an object's attributes and features regardless of its context whereas holistic processing is thought to involve greater attention to context and the relationship between objects. Studies have shown that East Asians attend more to the field and background context, and find it more difficult to focus on an object while ignoring the field in which it is embedded than European Americans, who attend more to salient, or focal objects (Nisbett et al. 2001; Nisbett and Miyamoto 2005). This finding has been replicated across a range of tasks such as change blindness (Masuda and Nisbett 2006) and the framed line test (Kitayama et al. 2003) and has also been supported with data from eye-tracking studies (Chua et al. 2005).

Experimental studies have also found self-construal style to result in differential processing of socioaffective information. For example, Masuda et al. (2008) found that judgment of facial expression derived from social context were used differentially for Japanese and Westerners. When viewing the emotions of a central face, Japanese tended to look at the surrounding faces more often and to incorporate information derived from the surrounding faces more than Westerners. Similarly, Goto et al. (2013) found that Asian Americans processed incongruities between foreground facial expressions and background affective scenes, as indexed by the N400. Together, these studies suggest that East Asians and Asian Americans tend to perceive facial emotion as embedded in contexts. We were particularly interested in the N400 as a measure of cognitive processing of socioaffective context. The N400 has previously been shown to be sensitive to cultural difference and inversely associated with semantic relatedness of social stimuli (e.g., Goto et al. 2013). Specifically, we sought to determine whether temporarily heightening the awareness of a particular cultural schema (e.g., independence or interdependence) through priming would result in cultural group differences in enhanced contextual processing of semantically congruent and incongruent socioaffective scenes.

A review of the neural priming literature also reveals that most neural priming studies have relied on priming Westernized Asians, a notable exception being Sui et al.'s (2013) comparison of Chinese and British participants. The finding from Sui et al. (2013) that monocultural samples also show an effect of cultural priming is consistent with literature showing the effects of primes on monoculturals across behavioral (e.g., Oyserman and Lee 2008) and neuroscience (e.g., Varnum et al. 2014) domains. To the extent that independent and interdependent orientations exist in some degree in all cultures, finding an effect of self-construal primes on monocultural samples is not surprising (Oyserman et al. 2002). However, researchers have yet to compare the strength of the effects of cultural primes on monocultural compared to bicultural samples. 
Given Hong et al. (2000) conceptualization of the dynamic constructivist approach to culture, a comparison of biculturals and monoculturals might be particularly interesting. They posit that biculturals, more than monoculturals, internalize two relatively strong cultural schemas and move between one cultural frame and another based on salient schemata in their daily lives. Thus, although monocultural Americans are expected to show some effect of cultural prime, biculturals in America should show greater influence of cultural priming on neural activity.

We predicted an ethnicity (Asian American, European American) by prime (interdependent, independent) by congruity condition (congruent, incongruent) interaction such that bicultural Asian Americans primed with interdependent values would show a greater N400 response for semantically incongruent socioaffective scenes than congruent scenes when compared to similarly primed monocultural European Americans, and that bicultural Asian Americans primed with independent values would show no difference in N400 response to incongruent and congruent social scenes, similar to independently primed European Americans. That is, the pattern of results for Asian Americans or biculturals under the interdependent prime should replicate the results of Asian American semantic processing of information reported in Goto et al. (2013). Under an independent prime, Asian Americans should semantically process information similarly to European Americans (also in Goto et al. 2013), and European American semantic processing is expected to be relatively invariant across prime due to their monoculturalism. Furthermore, these priming effects were expected to emerge in the temporal lobe, which is associated with semantic processing (see Lau et al. 2008; Van Petten and Luka 2006).

\section{Method}

Participants

Participants were 33 undergraduate East Asian American students, and 31 undergraduate European American students, aged 18-22 years, from the Claremont Colleges, a consortium of liberal arts colleges in southern California. Between 12 and $17 \%$ of students at these colleges were Pell grant recipients (representing the lower two income quintiles nationally). However, a majority of participants came from families that were predominantly upper middle income and college educated. All participants were right-handed, monoracial and had normal or corrected to normal vision. The Asian American participants self-identified as bicultural through the Suinn-Lew Asian Self-Identity Acculturation Scale (SL-ASIA; Suinn et al. 1987). The mean SL-ASIA score is $2.77(S D=0.43)$ for Asian American students. Additionally, we required all Asian American students to be 1.5 generations or greater (where 1.5 generations is defined as people who immigrated to the US before the age of 12). The sample of Asian American students comprised 10 1.5generation students, 11 second-generation students, 2 third-generation students, and 3 fourth-generation students. Participants were recruited from introductory psychology courses and Asian American organizations across the Claremont 
Colleges. They were compensated $\$ 20$ or given subject pool credit for the session. The research was approved by the Pomona College Institutional Review Board, and informed written consent was obtained from each participant prior to the experiment.

Materials and procedure

\section{Cultural priming}

To control for the effects of experimenter ethnicity, an Asian American experimenter tested participants in the interdependent prime condition and a European American experimenter tested participants in the interdependent prime condition. In addition to the experimenter and following the cultural priming procedures outlined by Chiao et al. (2009), there was a 10-min priming procedure consisting of two priming tasks, the Sumerian Warrior Story task (Trafimow et al. 1991) and a modified Similarities and Differences with Friends and Family task (SDFF task; Trafimow et al. 1991), both of which have been shown to reliably impact self-construal (Oyserman et al. 2002).

\section{Visual stimuli}

Stimuli were cartoon images of people created using My Avatar Editor (http://www. myavatareditor.com/). Background stimuli were constructed with 120 unique images consisting of a group of four figures. Each background stimulus was presented once for each self-construal priming condition. Target stimuli consisted of 40 unique focal figures presented in the middle of the surrounding background figures (see Fig. 1). Each focal figure appeared happy three times and sad three times for a total of six appearances. All of the figures had either a happy or sad facial expression. Because of the possibility for differences in how people interpret the faces of culturally familiar and unfamiliar faces (Elfenbein and Ambady 2002), we used Caucasian and Asian boys and girls. Congruent pairings consisted of background (i.e., surrounding) figures that were all expressing the same emotion as the focal figure (e.g., all figures have happy facial expressions). In incongruent pairings, the facial expression of background figures were not the same as the facial expression of the focal figure (e.g., happy background figures with sad focal face). In distracter pairings, the facial expressions of the focal and background figures were mixed (e.g., happy and sad facial expressions).

The stimuli were presented on a Dell 22-inch computer monitor centrally positioned at eye level $75 \mathrm{~cm}$ from the observer's eyes. The computer program E-Prime 2.0 (Psychology Software Tools Inc., Pittsburgh, PA) was used to program the presentation of the stimuli.

\section{Self-construal scale}

Developed by Singelis (1994), the 30-item self-construal scale includes 15 independent items (e.g., "I enjoy being unique and different from others in many 
(a)

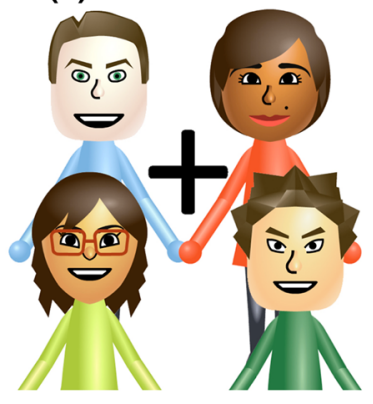

(c)

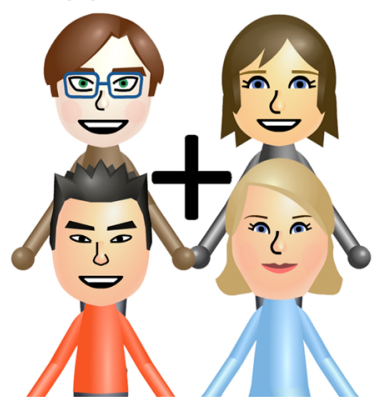

(b)

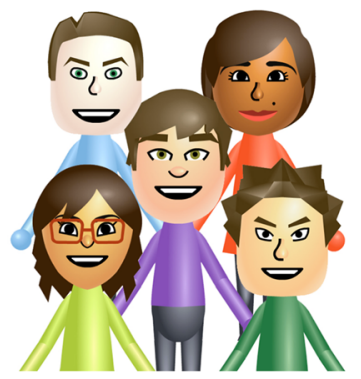

(d)

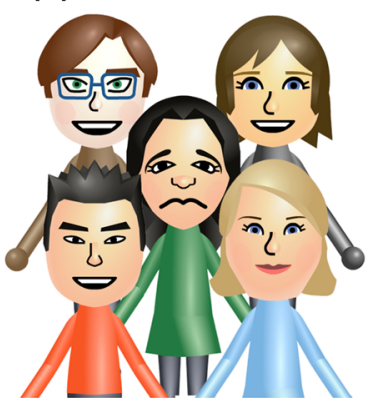

Fig. 1 Sample stimuli. Top row (a, b): sample congruent visual stimuli. First, the surrounding figures are presented (a). Then, a figure expressing the same facial expression as the surrounding figures is shown superimposed with the images of the surrounding figures $(\mathbf{b})$. Bottom row $(\mathbf{c}, \mathbf{d})$ : sample incongruent visual stimuli. First, the surrounding figures are presented (c). Then, a figure expressing a different facial expression as the surrounding figures is shown superimposed with the images of the surrounding figures (d)

respects") and 15 interdependent items (e.g., "I feel my fate is intertwined with the fate of those around me"). Each item was rated on a Likert scale from 1 (Strongly Disagree) to 7 (Strongly Agree).

Suinn-Lew Asian Self-Identity Acculturation (SL-ASIA) Scale

The SL-ASIA Scale (Suinn et al. 1992) is a 26-item scale measuring acculturation in Asian populations.

\section{Distracter task}

Participants were asked to complete a 20-piece online jigsaw puzzle (http://www.jigzone. com) of a scene of colored pencils as a distracter between the two prime conditions.

Procedure

By random assignment, participants were presented with either the independent or interdependent prime version of the Sumerian Warrior Story and SDFF tasks. After 
self-construal priming, participants performed the N400 facial expression task. Practice trials were followed by five blocks of 24 trials: 50 trials of which the focal and surrounding faces had congruent expressions, 50 trials of which the focal and surrounding faces had incongruent expressions, and 20 distractor trials. Between each block, subjects were allowed to take a break. Participants were presented with a fixation point ("+") in the center of the computer screen for a randomly determined interval between 500 and 1,500 ms. Then, a background image (with four figures) was presented for $600 \mathrm{~ms}$. This was followed by a focal figure superimposed upon the background for $600 \mathrm{~ms}$. The sequence of trials was randomized for each subject.

The orienting task for participants was to determine whether the focal figure was happy or sad. Participants were given two response boxes, one for each hand. If the focal figure was happy, they were instructed to press " 1 " simultaneously with both hands. If the focal object was sad, they were instructed to press " 2 ." If they were unsure, then they were instructed to press " 3 ." Reaction time and accuracy were recorded.

After the first prime condition, participants completed the jigsaw puzzle distracter task. Participants were then administered the other self-construal priming task, followed by the ERP affective facial expression task. The ethnicity of the experimenters was switched according to the self-construal prime. The order of the prime condition was counterbalanced across participants within each ethnic group. (Order of the prime condition did not interact with ethnic group or the N400 ERP task. Therefore, order of the prime condition was collapsed for subsequent analyses.) After the second round of primes, participants performed the affective facial expression task again. After the experimental procedure, East Asian Americans participants completed the SL-ASIA scale and all participants completed demographic questions and surveys.

\section{EEG acquisition}

The EEG was continuously recorded using Electrical Geodesics Inc. 256-channel Hydrocel Geodesic Sensor Net soaked in a potassium chloride saline solution (Electrical Geodesics Inc., Eugene, OR). The electrodes were Ag/AgCl-plated carbon-fiber pellets connected to a gold pin by a lead-shielded wire. The net was connected to a DC-coupled high impedance (200 M $\Omega$ ) Net Amps 300 amplifier. Analog voltages were amplified by a gain of 1,000 and a bandpass filter of $0.3-100 \mathrm{~Hz}$ was used during recording. Voltages were digitized with a 24-bit A/D converter at $250 \mathrm{~Hz}$. Recording electrodes were referenced to the subject's vertex electrode. The impedance for all the electrodes were kept below $50 \mathrm{k} \Omega$.

\section{ERP analysis}

NetStation 4.5 software was used to process the raw EEG data (Electrical Geodesics Inc., Eugene, OR). Raw data were filtered using a $30 \mathrm{~Hz}$ lowpass filter. ERPs were segmented beginning $100 \mathrm{~ms}$ prior to the onset of the superimposed focal face and ending 1,000 ms after its onset. Trials contaminated by ocular artifacts (greater than 
$70 \mu \mathrm{V}$ difference between eye channels) or more than five bad channels (100 $\mu \mathrm{V}$ difference between successive samples or reaching amplitudes of $200 \mu \mathrm{V}$ ) were excluded from the average. Participants with less than 25 artifact free trials in either the congruent or incongruent conditions for either prime were eliminated from analyses in the study. This resulted in the elimination of 7 East Asian American participants and 5 European American participants, leaving 26 East Asian American and 26 European American participants in the study. The EEG was averaged separately for the congruent and incongruent affective conditions within each priming condition.

Since the N400 consists of anterior and posterior scalp components (see Frishkoff et al. 2004), we separately averaged the N400 for anterior midline electrodes (\#15, Fz, \#26, and \#31), and centroposterior electrodes ( $\mathrm{Cz}, \# 81, \# 90$, and $\mathrm{Pz}$,$) . We$ calculated the adaptive mean amplitude, averaged across the electrodes and corresponding to $25 \%$ of the amplitude on either side of the peak negativity within a 250-500 ms temporal window. We used the adaptive mean in order to minimize the effect of noise, relative to using peak amplitude, and to better capture individual differences in the N400, relative to using mean amplitude across the entire temporal window (see Clayson et al. 2013).

\section{Source estimation}

A linear inverse minimum norm solution with standardized low-resolution brain electromagnetic tomography constraint (sLORETA) was used to estimate intracerebral current sources of the scalp potential (Pascual-Marqui 2002). We used a version of sLORETA implemented by EGI GeoSource 2.0 software (Electrical Geodesics Inc., Eugene, OR). A finite difference head (FDM) model was used for calculation of the lead field in relation to the head tissues. The FDM model was constructed from a subject (Colin27) who most closely resembled the Montreal Neurological Institute average head (MNI305) (Holmes et al., 1998). Conductivity values of the scalp, skull, CSF, and brain are $0.44,0.018,1.79$, and $0.25 \mathrm{~S} / \mathrm{ml}$, respectively. Source space consisted of 2,447 cortical voxels $(7 \mathrm{~mm})$ with three orthogonal orientations. Source estimates were performed on the grand averaged scalp data and superimposed upon the Colin27 MRI.

\section{Results}

\section{Self-construal orientation}

Retaining all items of the Singelis (1994) Self-Construal Scale (SCS) resulted in a Cronbach alpha of 0.77 for the independent subscale, and a Cronbach alpha of 0.76 for the interdependent subscale, suggesting moderately high reliability for both scales. East Asian Americans $(M=4.06, S D=0.50)$ were marginally more interdependent than European Americans $(M=3.81, S D=0.52)$ on the Singelis SCS, $t(50)=1.78, \quad p=.08$. However, European Americans $(M=4.17$, 
$S D=0.64)$ were not more independent than East Asian Americans $(M=4.22$, $S D=0.57)$ on the Singelis SCS, $t(50)<1$, ns.

Manipulation check

To evaluate the effectiveness of the priming procedures, a manipulation check was performed on participant responses to the modified SDFF task (see Chiao et al. 2009). Two independent raters read and rated the essays. Raters were blind to the essay prompts and essays were presented in a random order. Essays were rated on how much they thought the authors valued their independence or their interdependence using a Likert scale from 1 (mostly independent) to 5 (mostly interdependent). The criteria for independence included emphasizing achievement of individual goals, personal traits, and differences from friends and family. The criteria for interdependence included emphasizing fulfillment of social expectations, relational roles, and similarity to friends and family. Cohen's kappa indicated high inter-rater reliability for both the interdependent prime (0.97) and independent prime (0.94) conditions. There was a main effect of priming on ratings, $F(1$, $48)=115.25, p<.001$, such that ratings in the interdependent priming condition $(M=3.80, S D=1.21)$ were higher (more interdependent) than ratings in the independent priming condition $(M=1.73, S D=0.89)$, indicating the prime was successful in eliciting the appropriate behavioral response from participants.

There was a marginally significant main effect of ethnicity on ratings, $F(1$, $48)=3.07, p=.09$. Asian American participants $(M=2.94, S D=0.15)$ had higher ratings for both primes compared to European American participants $(M=2.56, S D=0.15)$, suggesting slightly more interdependent responses across priming conditions.

\section{Behavioral analyses}

In order to determine group or condition differences in accuracy and reaction time to the facial affective decision task, separate ethnic group (Asian American, European American) $\times$ prime (interdependent, independent) repeated measures ANOVAs were conducted. One European American was omitted due to missing data. Therefore, analyses were conducted on 25 European Americans. For accuracy, there was no main effect of either ethnic group, $F(1,49)=2.21$, ns or priming condition, $F(1,49)<1$, ns, nor was there an interaction, $F(1,49)<1$, ns. Similarly, for reaction time there was no main effect of either ethnic group, $F(1,49)<1$, ns or priming condition, $\mathrm{F}(1,49)=1.7$, ns, nor was there an interaction, $F(1,49)<1$, ns. See Tables 1 and 2 for means and standard deviations.

Electrophysiological analyses

\section{Scalp voltage}

In order to investigate the relationship among self-construal priming, ethnic group, and facial expression incongruity on the N400, we conducted an ethnic group (Asian 
American, European American) $\times$ prime (interdependent, independent $) \times$ congruity condition repeated measures ANOVA separately for the anterior and posterior midline electrodes (see Table 3; Figs. 2, 3).

For the anterior midline electrodes, there was a main effect of congruity, $F(1$, $50)=8.37, p=.006$, such that the $\mathrm{N} 400$ was more negative for the incongruent than congruent trials. Consistent with our hypothesis, there was a three-way interaction, $F(1,50)=4.53, p=.04$. For Asian Americans, there was a significant priming $\times$ congruity interaction, $F(1,25)=4.98, p=.04$, such that following the interdependent prime, they had a significantly larger N400 to incongruent than congruent facial expressions, $t(25)=3.11, p=.005$, whereas there was no significant difference in the $\mathrm{N} 400$ between the incongruent and congruent conditions following the independent prime, $t(25)<1$, ns. In contrast, for the European Americans, there was no prime $\times$ congruity interaction, $F(1,25)<1$, ns nor was there a main effect of congruity, $F(1,25)=2.60$, ns. There was, however, a trend for greater negativity of the $\mathrm{N} 400$ following the interdependent prime, $F(1$, 25) $=3.18, p=.09$.

Furthermore, there was no priming $\times$ ethnic group interaction, $F(1,50)<1$, ns, ethnic group $\times$ congruity interaction, $F(1,50)<1$, ns, or priming $\times$ congruity interaction, $F(1,50)<1$, ns. There were also no main effects of priming condition, $F(1,50)=2.55$, ns or ethnic group, $F(1,50)=2.56$, ns. Therefore, for the anterior midline electrodes, only the Asian Americans showed an N400 incongruity effect, and this was found only following the interdependent prime.

For the posterior midline electrode positions, there was a non-significant trend for greater N400 negativity following the independent relative to interdependent priming condition, $F(1,50)=3.14, p=.08$. There was also a prime $\times$ ethnic group interaction, $F(1,50)=5.55, p=.02$, such that Asian Americans displayed increased negativity following the independent prime compared to the interdependent prime. However, there was not a main effect of congruity, $F(1,50)<1$, ns, nor was there an ethnic group $\times$ congruity interaction, $F(1,50)<1$, ns, priming $\times$ congruity interaction, $F(1,50)=2.20$, ns, or priming $\times$ ethnic group $\times$ congruity interaction, $F(1,50)<1$, ns.

\section{Source estimation}

The sLORETA source solution localized strongest activity to the medial temporal lobe area (primarily the left parahippocampal and fusiform gyrus). The greatest activity was found for the Asian Americans during the interdependent prime condition in the left parahippocampal gyrus during the 350-450 ms temporal window (see Fig. 4). A composite dipole was calculated consisting of the voxel of highest intensity and adjacent dipoles showing $80 \%$ or greater values, which included 7 adjacent dipoles. The resulting source current values were entered into an ethnic group by congruity condition repeated measures ANOVA for each priming condition.

For the interdependent prime condition, consistent with the scalp analysis, there was a marginally significant ethnic group by congruity condition interaction, $F(1$, $50)=3.01, p=.09$. Asian Americans had a significantly larger current in the left 
Table 1 Accuracy of facial emotion judgment

\begin{tabular}{lll}
\hline Self-construal priming & $\begin{array}{l}\text { European Americans }(n=25) \\
M(S D)\end{array}$ & $\begin{array}{l}\text { Asian Americans }(n=26) \\
M(S D)\end{array}$ \\
\hline Independent & $0.97(0.03)$ & $0.94(0.08)$ \\
Interdependent & $0.96(0.04)$ & $0.95(0.08)$ \\
\hline
\end{tabular}

Table 2 Mean reaction times for facial emotion judgment (in milliseconds)

\begin{tabular}{lll}
\hline Self-construal priming & $\begin{array}{l}\text { European Americans }(n=25) \\
M(S D)\end{array}$ & $\begin{array}{l}\text { Asian Americans }(n=26) \\
M(S D)\end{array}$ \\
\hline Independent & $520.27(79.01)$ & $534.46(120.18)$ \\
Interdependent & $547.01(118.90)$ & $544.58(105.56)$ \\
\hline
\end{tabular}

Table 3 N400 mean amplitude (in $\mu \mathrm{V}$ )

\begin{tabular}{lll}
\hline Self-construal priming & $\begin{array}{l}\text { European Americans }(n=26) \\
M(S D)\end{array}$ & $\begin{array}{l}\text { Asian Americans }(n=26) \\
M(S D)\end{array}$ \\
\hline Independent & & \\
Anterior N400 & & \\
$\quad$ Congruent condition & $-0.80(3.04)$ & $-0.11(3.43)$ \\
$\quad$ Incongruent condition & $-1.34(3.26)$ & $-0.28(2.81)$ \\
Posterior N400 & $0.03(2.60)$ & $-0.85(2.11)$ \\
$\quad$ Congruent condition & $-0.48(1.99)$ & $-1.21(3.03)$ \\
Incongruent condition & & \\
Interdependent & $-1.94(2.73)$ & $-0.07(3.42)$ \\
Anterior N400 & $-2.05(2.68)$ & $-1.18(3.14)$ \\
Congruent condition & & \\
Incongruent condition & $-0.42(2.25)$ & $-0.04(1.58)$ \\
Posterior N400 & $-0.36(2.38)$ & $0.28(2.33)$ \\
Congruent condition & &
\end{tabular}

medial temporal lobe source during the incongruent compared to the congruent conditions, $t(25)=2.17 ; p=.04$. In contrast, for the European Americans there was no difference in current between the two congruity conditions, $t(25)<1$, ns. There was no main effect of congruity, $F(1,50)=2.40$, ns, or ethnic group, $F(1$, 50) $<1$, ns.

As expected, for the independent prime condition, there was no main effect of congruity condition, $F(1,50)<1$; ns or ethnic group, $F(1,50)=1.49$, ns, nor was there an interaction between the two, $F(1,50)=2.20$, ns. 


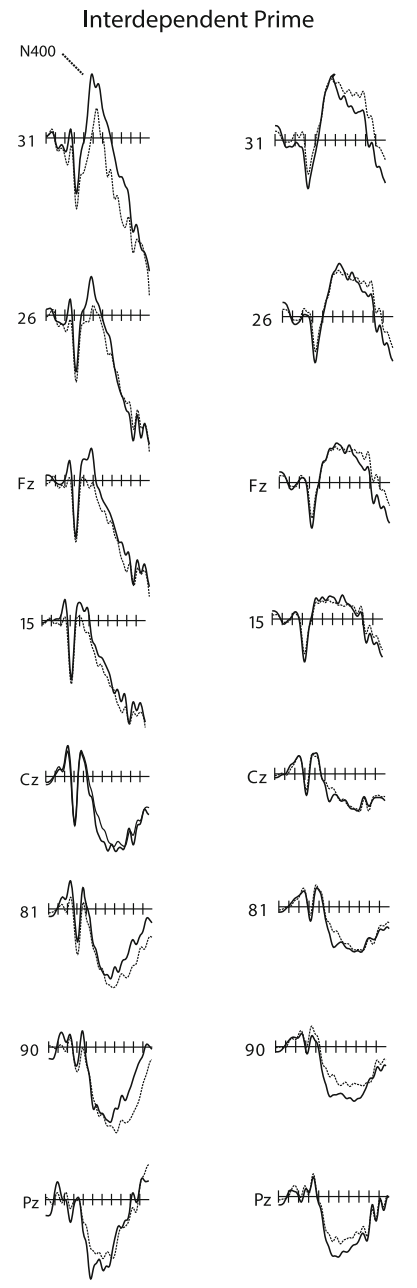

Asian Americans

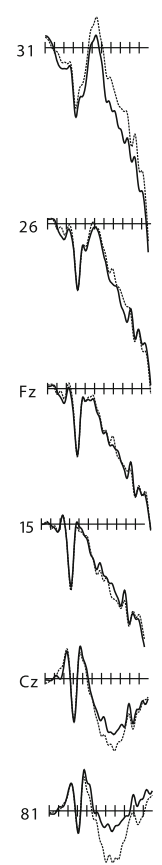

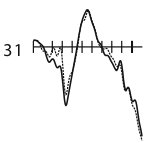

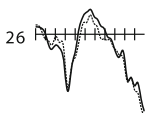

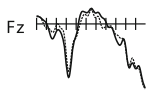

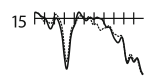

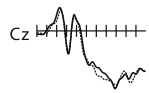<smiles>FC(F)=C1CCCC1C(F)Br</smiles>

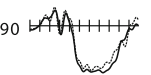

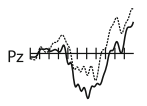

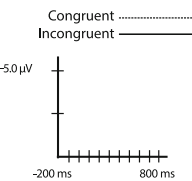

European Americans

Fig. 2 Event-related potentials for Asian Americans and European Americans primed with interdependent and independent self-construals during the congruent (light dotted line) and incongruent (dark line) conditions for midline electrodes. Voltage is plotted as a function of time $200 \mathrm{~ms}$ pre-stimulus onset to $1,600 \mathrm{~ms}$ post-stimulus onset. Asian Americans displayed a greater N400 response to the incongruent condition following the interdependent priming, but not following independent priming. In contrast, European Americans did not show an N400 incongruity effect following either priming condition

\section{Discussion}

We investigated the effects of cultural priming on the semantic processing of affectively-valenced social context. Bicultural Asian American and monocultural European American participants were primed with independent or interdependent 

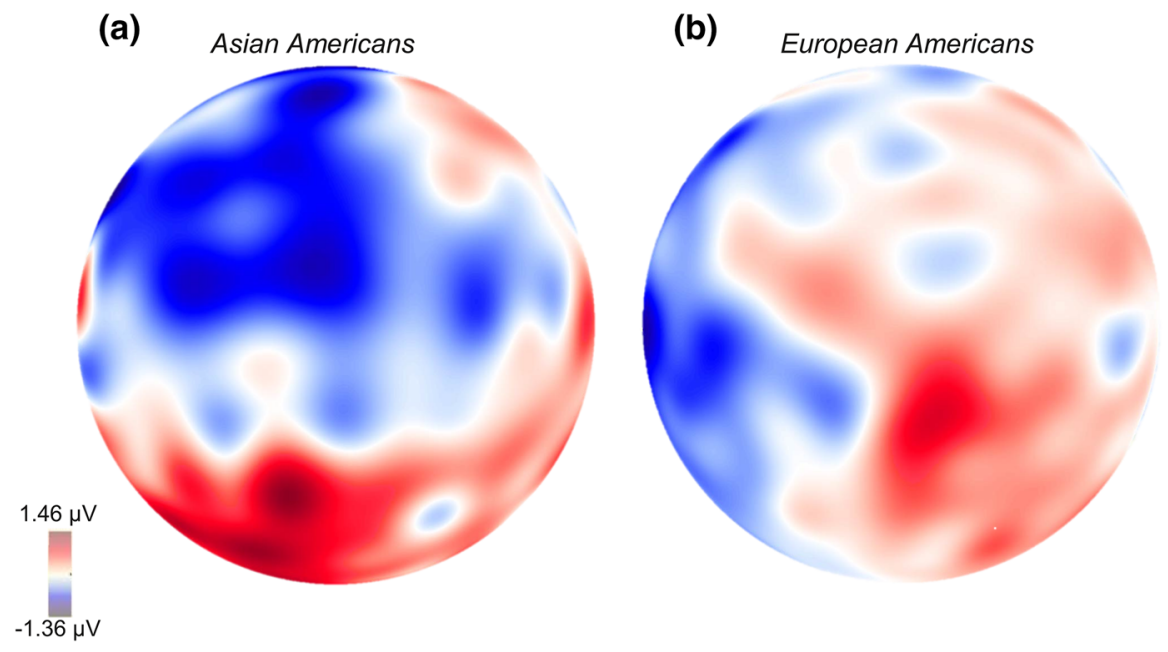

Fig. 3 Scalp topography of voltage difference between the incongruent and congruent conditions for the European Americans (left scalp topography) and for the Asian Americans (left scalp topography) at $375 \mathrm{~ms}$ post onset of central figure. Greater negativity for the incongruent condition is indicated by blue coloring. Notice the greater N400 during the incongruent condition for Asian Americans along the left anterior electrode sites. Color coded scale is in $\mu \mathrm{V}$. (Color figure online)

self-construals before viewing positive or negative facial expressions of four background figures, followed by either an affectively congruent or incongruent focal face. Resulting N400 s were measured to infer the amount of semantic processing of the relationship between the background facial expressions and focal facial expression. As hypothesized, following the interdependent priming condition, there was a greater N400 incongruity effect for Asian Americans than for European Americans. Subsequent source analyses indicated that left medial temporal lobe activity contributed to the observed N400 effect in Asian Americans during the interdependent prime condition. In contrast, independent priming abolished the N400 incongruity effect for both ethnic groups. This study extends our understanding of the cultural priming literature to include semantic processing of social affective information. Our ERP results also contribute to the extant N400 literature by demonstrating that semantic processing effects are not only culturally sensitive, but can also be influenced within the same individual through selfconstrual priming.

These findings are consistent with recent priming studies and importantly extend the robustness of the range of priming beyond the processing of self and others. For example, Chiao et al. (2009) found that Asian Americans showed increased medial prefrontal cortex activity during contextual (e.g., 'When talking to my mother, I am truthful') relative to general (e.g., 'In general, I am truthful') self-judgments after interdependent self-construal priming and the opposite effect during independent self-construal priming. Similarly, Ng et al. (2010) found that under Chinese, but not Western, cultural priming, Westernized bicultural Chinese showed overlap of neural representations of the self and a close other in the medial prefrontal cortex. 


\section{(a)}

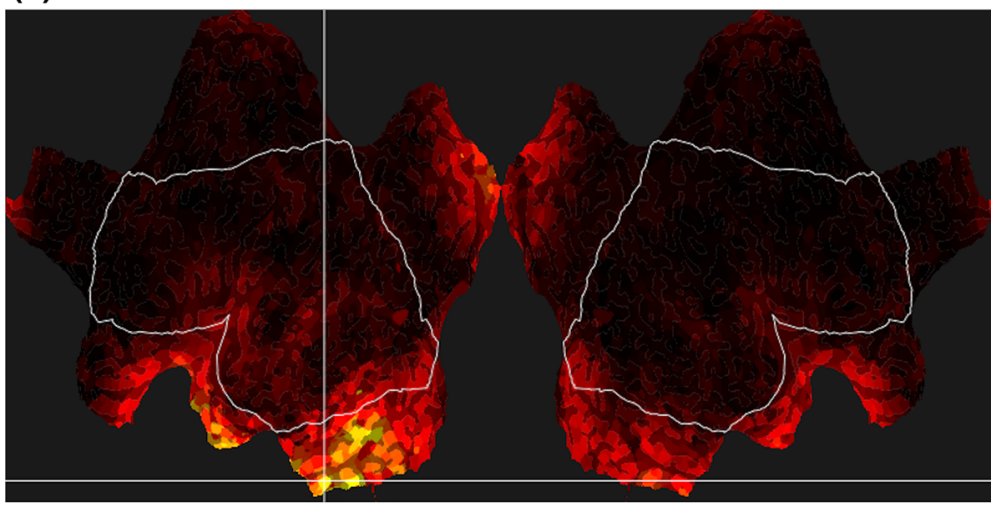

(b)

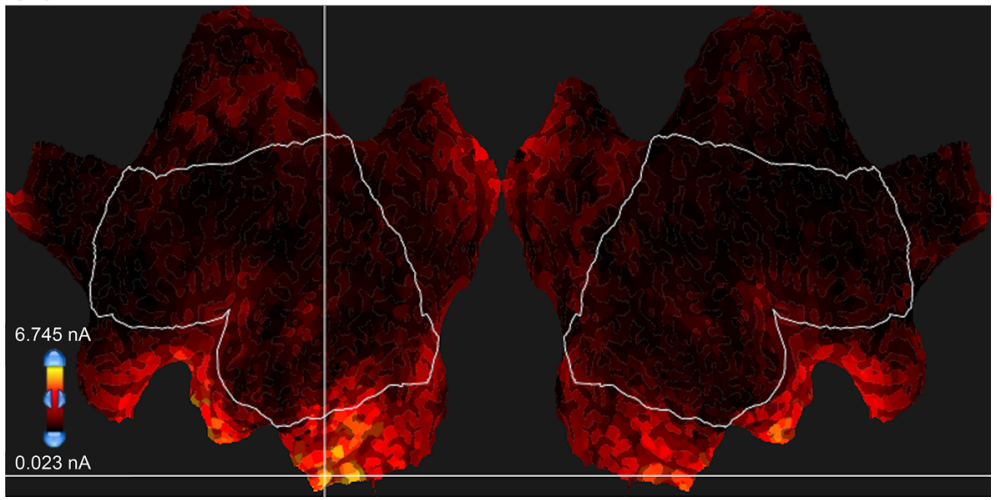

Fig. 4 Cortical flatmap of source imaging analysis at $375 \mathrm{~ms}$. Post onset of central figure. a The difference in current between the incongruent and congruent conditions for the Asian Americans, and $\mathbf{b}$ the results for the European Americans. The left side of each panel displays the left hemisphere and the right side of each panel displays the right hemisphere. Peak intensity occurred in the left parahippocampus gyrus, corresponding to Brodmann Area 35 (Tailarach coordinates -24, -25, -27).

The current study's findings are consistent with Hong et al.'s (2000) dynamic constructivist approach to culture in which biculturals move between different cognitive frames (i.e., frame-switch) based on the most accessible cultural schemas. Within this framework, Asian Americans have access to both East Asian holistic processing styles and European American analytic processing styles (Nisbett and Miyamoto 2005). In our study, when primed with interdependent self-construals, Asian Americans had a more context dependent (holistic) processing style, with greater semantic processing of the relationship between the focal and background figures' facial expressions. In contrast, when primed with independent selfconstruals, Asian Americans had a more context independent (analytic) processing style, with the foreground facial expression processed independently of the background figures' facial expressions. When related to Goto et al.'s (2013) specific findings, biculturals semantically process information like the Asian American 
sample when primed interdependently, but process information like the European American sample when primed independently.

Interestingly, this appears somewhat inconsistent with the culture-as-situatedcognition model, which suggests that both independent and interdependent selfconstruals exist in all cultures but that one is more chronically accessible (Oyserman et al. 2009; Sui et al. 2013). Under this model, the independent self-construal is more accessible than the interdependent self-construal for Western monocultural individuals. However, when contextual cues prime interdependent self-construals, Westerners should display responses typical of interdependent self-construals. Indeed, priming studies have found behavioral and neural patterns in monoculturals consistent with this model (e.g., Alter and Kwan 2009; Hong et al. 2000; Sui et al. 2013).

Somewhat surprisingly, among European American participants, we did not find changes in neural activity reflective of holistic (i.e., East Asian) processing under the interdependent prime. Given that our prime manipulation relied on established techniques (Oyserman et al. 2002) and our manipulation check suggested that the prime was effective, it is not entirely clear as to why we did not see an effect of the interdependent prime on European Americans at the neural level. It is possible that for European Americans, the effect of the interdependent prime weakened between the manipulation check and the start of the N400 task given the broader, chronic independent context (i.e., at a U.S. college) in which the experiment took place. Similar to our study, Wang et al. (2013) found less priming effects (i.e., less priming task-induced activity) among independently primed Chinese participants in the dorsal medial prefrontal cortex compared to interdependently primed Chinese participants. However, our data revealed no difference across groups in self reported independence. The overall pattern of effects may be due to a culture-as-situatedcognition model for biculturals, but not for monoculturals.

Furthermore, these data suggests that the interdependent prime is redundant with the default, holistic style for Asian Americans and provides support for the chronic culture priming mismatch hypothesis. In several studies by Han and colleagues, the prime that is most mismatched with the chronic culture will be most effective (i.e., interdependent prime for Western Chinese; e.g., Ng et al. 2010; Sui and Han 2007; Sui et al. 2013). Asian Americans are chronically exposed to Asian primes most likely through family and friend socialization. Our data are consistent with the chronic culture priming mismatch hypothesis by revealing the strength of the independent prime for our Asian American sample. Clearly, more research is needed to clarify the role of monoculturalism, interdependence/independence, prime, and task in the priming of monoculturals.

The results of the source estimation analyses suggest that cultural priming is affecting left temporal lobe semantic processing mechanisms. Our source estimation results are consistent with a multitude of studies localizing the N400 to the temporal lobe area (see Lau et al. 2008; Van Petten and Luka 2006 for reviews), however there are generators outside of this area as well. Although the N400 is sensitive to cultural priming in biculturals, this does not necessarily mean that cultural priming is directly affecting temporal lobe semantic processing. Instead, it is possible that cultural priming affects early processes, which are reflected in later semantic 
processing. For example, Lin et al. (2008) have demonstrated that cultural priming modulated the early visual P1 ERP, associated with local/global attentional processes. This suggests that cultural priming could affect the scope of attention, which would subsequently influence the sensitivity to processing affective incongruity among facial expressions. More research is needed to better understand the extent to which cultural priming influences the range of neurocognitive processes.

Additionally, behavioral measures of accuracy and reaction time were not sensitive to within or between-group differences. This is consistent with the cultural neuroscience literature and attests to the sensitivity of neural measures in investigations of culture. In particular, neuroscience techniques may be better suited to detect subtle or unconscious aspects of cognition and can inform theories about the process or mechanisms behind cultural differences in cognition (Goto et al. 2010, 2013). To date, we know surprisingly little about the neural correlates of cultural priming, especially in multicultural individuals (Wang et al. 2013). Better understanding of the interrelationships between behavioral, cognitive, and neural effects of priming is an area ripe for future exploration.

Interestingly, we found no significant differences in independent or interdependent self-construal across Asian Americans and European Americans in our sample. Although prior studies have typically established differences in self-construal styles (Heine 2001), other meta-analyses have suggested no consistent cultural differences between East Asian and European American groups (e.g., Oyserman et al. 2002). Heine et al. (2002) reconcile these findings by suggesting a reference-group effect for subjective Likert scales. In our sample, a European American participant who strongly endorses a question regarding her or his interdependence may be referring to a lower threshold of interdependence compared to an Asian American participant. As such, reference-group effects may have masked differences in self-construal styles between Asian Americans and European Americans.

Given that the samples in this study were drawn from college students, the generalizability of the findings may be limited. College students may have cultural values that differ from the population because they have a high level of education and higher socioeconomic background (Henrich et al. 2010). Although studies have not found evidence for the magnitude of cultural differences between college students and adults, future studies should include samples of more diverse populations. A second limitation of the current study is the lack of a no-prime condition. However, in a similar N400-paradigm study, Goto et al. (2013) found Asian Americans showed greater N400 to affectively incongruent scenes compared to affectively congruent scenes while European Americans showed no difference. We would expect the same findings in the current sample. Our bicultural versus monocultural comparisons involved Asian Americans and European Americans. Other comparison groups are also warranted, namely Asian monoculturals and Caucasian biculturals.

The present study's focus on Asian Americans is particularly relevant given that Asians are the largest and fastest-growing immigrant group in the U.S. (The Pew Research Center 2012). As multiculturalism becomes more prevalent with increasing globalization, it will become even more important to understand how 
the brain adapts to diverse cultural values. Here, our findings begin to suggest a mechanism for how dynamic social information is processed in multicultural individuals. In sum, our study furthers understanding of cultural differences in cognition by suggesting semantic processing of social relationships as one possible mechanism for facilitating frame switching (Hong et al. 2000). This study importantly contributes to the knowledge of how situated cultural context affects processing of social phenomenon at the levels of behavior, cognition, and the brain.

Acknowledgments This research was funded by the Shanahan Student-Directed Research Funds at Harvey Mudd College.

Conflict of interest The authors declare that they have no conflict of interest.

Ethical standard This study has been approved by the Pomona College Institutional Review Board and has therefore been performed in accordance with the ethical standards in the 1964 Declaration of Helsinki and its later amendments.

\section{References}

Alter, A., \& Kwan, V. (2009). Cultural sharing in a global village: Evidence for extracultural cognition in European Americans. Journal of Personality and Social Psychology, 96, 742-760. doi:10.1037/ a0014036.

Berry, J. W. (1980). Acculturation as varieties of adaptation. In A. M. Padilla (Ed.), Acculturation: Theory, models and some new findings (pp. 9-25). Boulder, CO: Westview.

Briley, D. A., \& Wyer, R. S. (2002). The effects of group membership salience on the avoidance of negative outcomes: Implications for social and consumer decisions. Journal of Consumer Research, 29, 400-415. doi:10.1086/344426.

Chentsova-Dutton, Y. E., \& Tsai, J. L. (2010). Self-focused attention and emotional reactivity: The role of culture. Journal of Personality and Social Psychology, 98, 507-519. doi:10.1037/a0018534.

Chiao, J. Y., Harada, T., Komeda, H., Li, Z., Mano, Y., Saito, D., Parrish, T. B., Sadato, N., \& Iidaka, T. (2009). Dynamic cultural influences on neural representations of the self. Journal of Cognitive Neuroscience. doi:10.1162/jocn.2009.21192.

Chua, H. F., Boland, J. E., \& Nisbett, R. E. (2005). Cultural variation in eye movements during scene perception. Proceedings of the National Academy of Sciences, 102, 12629-12633. doi:10.1073/pnas. 0506162102.

Clayson, P. E., Baldwin, S. A., \& Larson, M. J. (2013). How does noise affect amplitude and latency measurement of event-related potentials (ERPs)? A methodological critique and simulation study. Psychophysiology, 50, 174-186. doi:10.1111/psyp.12001.

Elfenbein, H. A., \& Ambady, N. (2002). On the universality and cultural specificity of emotion recognition: A meta-analysis. Psychological Bulletin, 128, 203-235. doi:10.1037/0033-2909.128.2. 203.

Frishkoff, G. A., Tucker, D. M., Davey, C., \& Scherg, M. (2004). Frontal and posterior sources of eventrelated potentials in semantic comprehension. Cognitive Brain Research, 20, 329-354. doi:10.1016/ S0926-6410\%2804\%2900060-6.

Goto, S. G., Ando, Y., Huang, C., Yee, A., \& Lewis, R. S. (2010). Cultural differences in the visual processing of meaning: Detecting incongruities between background and foreground objects using the N400. Social Cognitive and Affective Neuroscience, 5, 242-253. doi:10.1093/scan/nsp038.

Goto, S. G., Yee, A., Lowenberg, K., \& Lewis, R. S. (2013). Cultural differences in sensitivity to social context: Detecting affective incongruity using the N400. Social Neuroscience, 8, 63-74. doi:10. 1080/17470919.2012.739202. 
Harada, T., Li, Z., \& Chiao, J. Y. (2010). Differential dorsal and ventral medial prefrontal representations of the implicit self modulated by individualism and collectivism: An fMRI study. Social Neuroscience, 5, 257-271. doi:10.1080/17470910903374895.

Heine, S. J. (2001). Self as cultural product: An examination of East Asian and North American selves. Journal of Personality, 69, 881-906. doi:10.1111/1467-6494.696168.

Heine, S. J., Lehman, D. R., Peng, K., \& Greenholtz, J. (2002). What's wrong with cross-cultural comparisons of subjective Likert scales? The reference-group effect. Journal of Personality and Social Psychology, 82, 903-918. doi:10.1037/0022-3514.82.6.903.

Henrich, J., Heine, S. J., \& Norenzayan, A. (2010). The weirdest people in the world? Behavioral and Brain Sciences, 33, 61-83. doi:10.1017/S0140525X0999152X.

Holmes, C. J., Hoge, R., Collins, L., Woods, R., Toga, A. W., \& Evans, A. C. (1998). Enhancement of MR images using registration for signal averaging. Journal of Computer Assisted Tomography, 22, 324-333. doi:10.1097/00004728-199803000-00032.

Hong, Y., Morris, M. W., Chiu, C., \& Benet-Martínez, V. (2000). Multicultural minds: A dynamic constructivist approach to culture and cognition. American Psychologist, 55, 709-720. doi:10.1037/ 0003-066X.55.7.709.

Kitayama, S., Duffy, S., Kawamura, T., \& Larsen, J. T. (2003). Perceiving an object and its context in different cultures: A cultural look at new look. Psychological Science, 14, 201-206. doi:10.1111/ 1467-9280.02432.

Lau, E. F., Phillips, C., \& Poeppel, D. (2008). A cortical network for semantics: (De) constructing the N400. Nature Reviews Neuroscience, 9, 920-933. doi:10.1038/nrn2532.

Lee, R. M., Yoon, E., \& Liu-Tom, H. T. (2006). Structure and measurement of acculturation/ enculturation for Asian Americans using the ARSMA-II. Measurement and Evaluation in Counseling and Development, 39, 42-55.

Lin, Z., Lin, Y., \& Han, S. (2008). Self-construal priming modulates visual activity underlying global/ local perception. Biological Psychology, 77, 93-97. doi:10.1016/j.biopsycho.2007.08.002.

Markus, H. R., \& Hamedani, M. G. (2007). Sociocultural psychology: The dynamic interdependence among self systems and social systems. In S. Kitayama \& D. Cohen (Eds.), Handbook of cultural psychology (pp. 3-39). New York, NY: Guilford Press.

Markus, H. R., \& Kitayama, S. (1999). Culture and the self: Implications for cognition, emotion, and motivation. In R. F. Baumeister \& R. F. Baumeister (Eds.), The self in social psychology: Culture and the self: Implications for cognition, emotion, and motivation (pp. 339-371). New York, NY: Psychology Press.

Masuda, T., Ellsworth, P. C., Mesquita, B., Leu, J., Tanida, S., \& Van de Veerdonk, E. (2008). Placing the face in context: Cultural differences in the perception of facial emotion. Journal of Personality and Social Psychology, 94, 365-381. doi:10.1037/0022-3514.94.3.365.

Masuda, T., \& Nisbett, R. E. (2006). Culture and change blindness. Cognitive Science: A Multidisciplinary Journal, 30, 381-399. doi:10.1207/s15516709cog0000_63.

Ng, S. H., Han, S., Mao, L., \& Lai, J. C. (2010). Dynamic bicultural brains: fMRI study of their flexible neural representation of self and significant others in response to culture primes. Asian Journal of Social Psychology, 13, 83-91. doi:10.1111/j.1467-839X.2010.01303.x.

Nisbett, R. E., \& Miyamoto, Y. (2005). The influence of culture: Holistic versus analytic perception. Trends in Cognitive Sciences, 9, 467-473. doi:10.1016/j.tics.2005.08.004.

Nisbett, R. E., Peng, K., Choi, I., \& Norenzayan, A. (2001). Culture and systems of thought: Holistic versus analytic cognition. Psychological Review, 108, 291-310. doi:10.1037/0033-295X.108.2.291.

Oishi, S. (2000). Goals as cornerstones of subjective well-being: Linking individuals with cultures. In E. F. Diener \& E. M. Suh (Eds.), Subjective well-being across cultures (pp. 87-112). Cambridge, MA: MIT Press.

Oyserman, D., Coon, H. M., \& Kemmelmeier, M. (2002). Rethinking individualism and collectivism: Evaluation of theoretical assumptions and meta-analyses. Psychological Bulletin, 128, 3-72. doi:10. 1037/0033-2909.128.1.3.

Oyserman, D., \& Lee, S. W. S. (2008). Does culture influence what and how we think? effects of priming individualism and collectivism. Psychological Bulletin, 134, 311-342. doi:10.1037/0033-2909.134. 2.311 .

Oyserman, D., Sorensen, N., Reber, R., \& Chen, S. (2009). Connecting and separating mind-sets: Culture as situated cognition. Journal of Personality and Social Psychology, 97, 217-235. doi:10.1037/ a0015850. 
Pascual-Marqui, R. D. (2002). Standardized low-resolution brain electromagnetic tomography (sLORETA): Technical details. Methods and Findings in Experimental and Clinical Pharmacology, 24, $5-12$.

Singelis, T. M. (1994). The measurement of independent and interdependent self-construals. Personality and Social Psychology Bulletin, 20, 580-591. doi:10.1177/0146167294205014.

Sui, J., \& Han, S. (2007). Self-construal priming modulates neural substrates of self-awareness. Psychological Science, 18, 861-866. doi:10.1111/j.1467-9280.2007.01992.x.

Sui, J., Hong, Y. Y., Liu, C. H., Humphreys, G. W., \& Han, S. (2013). Dynamic cultural modulation of neural responses to one's own and friend's faces. Social Cognitive and Affective Neuroscience, 8 , 326-332. doi:10.1093/scan/nss001.

Suinn, R. M., Rickard-Figueroa, K., Lew, S., \& Vigil, P. (1987). The Suinn-Lew Asian Self-Identity Acculturation Scale: An initial report. Educational and Psychological Measurement, 47, 401-407. doi: $10.1177 / 0013164487472012$.

Tadmor, C. T., Tetlock, P. E., \& Peng, K. (2009). Acculturation strategies and integrative complexity the cognitive implications of biculturalism. Journal of Cross-Cultural Psychology, 40, 105-139. doi:10. $1177 / 0022022108326279$.

The Pew Research Center. (2012, July 12). The Rise of Asian Americans. Retrieved November 28, 2012, from http://www.pewsocialtrends.org/files/2012/06/SDT-The-Rise-of-Asian-Americans-Full-Report.pdf.

Trafimow, D., Triandis, H. C., \& Goto, S. G. (1991). Some tests of the distinction between the private self and the collective self. Journal of Personality and Social Psychology, 60, 649-655. doi:10.1006/ jesp. 1356.

Triandis, H. C. (2000). Dialectics between cultural and cross-cultural psychology. Asian Journal of Social Psychology, 3, 185-195. doi:10.1111/1467-839X.00063.

Triandis, H. C., McCusker, C., \& Hui, C. H. (1990). Multimethod probes of individualism and collectivism. Journal of Personality and Social Psychology, 59, 1006-1020. doi:10.1037/00223514.59.5.1006.

Van Petten, C., \& Luka, B. J. (2006). Neural localization of semantic context effects in electromagnetic and hemodynamic studies. Brain and Language, 97, 279-293. doi:10.1016/j.band1.2005.11.003.

Varnum, M. E., Shi, Z., Chen, A., Qiu, J., \& Han, S. (2014). When "Your" reward is the same as "My" reward: Self-construal priming shifts neural responses to own vs. friends' rewards. NeuroImage, 87, 164-169. doi:10.1016/j.neuroimage.2013.10.042.

Wang, C., Oyserman, D., Liu, Q., Li, H., \& Han, S. (2013). Accessible cultural mind-set modulates default mode activity: Evidence for the culturally situated brain. Social Neuroscience, 8, 203-216. doi:10.1080/17470919.2013.775966.

Yoon, E., Langrehr, K., \& Ong, L. (2011). Content analysis of acculturation research in counseling and counseling psychology: A 22-year review. Journal of Counseling Psychology, 58, 83-96. doi:10. 1037/a0021128. 